\title{
The role of automated compression devices in out-of- and in- hospital cardiac arrest. Can we spare rescuers' hands?
}

\author{
George Latsios, ${ }^{1}$ Marianna Leopoulou,,${ }^{1,2}$ Andreas Synetos, ${ }^{1}$ Antonis Karanasos, ${ }^{1}$ Eleni Melidi, ${ }^{1}$ \\ Kostas Toutouzas, ${ }^{1}$ Kostas Tsioufis ${ }^{1}$
}

${ }^{1} 1$ st Department of Cardiology, Medical School, National and Kapodistrian University of Athens, Hippokration General Hospital, Athens; ${ }^{2}$ Cardiology Department, Elpis Hospital, Athens, Greece

\begin{abstract}
Research regarding the use of mechanical compressions in the setting of a cardiac arrest, either outside of or inside the hospital environment has produced mixed results. The debate whether they can replace manual compressions still remains. The aim of this review is to present current literature contemplating the application
\end{abstract}

Correspondence: Dr George Latsios, Vas Sofias 114, 11527 Athens, Greece.

E-mail: glatsios@gmail.com

Key words: Cardiac arrest; mechanical compressions; manual compressions.

Contributions: GL: review concept and design, acquisition of the data, analysis and interpretation of the data, drafting of the manuscript, critical revision of the manuscript for important intellectual content; ML: review concept and design, acquisition of the data, analysis and interpretation of the data, drafting of the manuscript, critical revision of the manuscript for important intellectual content; AS: acquisition of the data, analysis and interpretation of the data, critical revision of the manuscript for important intellectual content; AK: acquisition of the data, analysis and interpretation of the data, critical revision of the manuscript for important intellectual content; EM: acquisition of the data, analysis and interpretation of the data, critical revision of the manuscript for important intellectual content; KT: analysis and interpretation of the data, critical revision of the manuscript for important intellectual content; KT: analysis and interpretation of the data, critical revision of the manuscript for important intellectual content.

Conflicts of interest: None. This work was not supported by any grant.

Availability of data and materials: All data generated or analyzed during this study are included in this published article.

Ethics approval and consent to participate: Not applicable.

Informed consent: Not applicable.

Received for publication: 24 November 2020.

Revision received: 28 February 2021.

Accepted for publication: 26 March 2021.

This work is licensed under a Creative Commons Attribution 4.0 License (by-nc 4.0).

(C) Copyright: the Author(s), 2021

Licensee PAGEPress, Italy

Emergency Care Journal 2021; 17:9525

doi:10.4081/ecj.2021.9525 of mechanical compressions in both settings, data comparing them to manual compressions as well as current guidelines regarding their implementation in everyday clinical use. Currently, their implementation in the resuscitation protocol seems to benefit the victims of an in-hospital cardiac arrest rather than the victims that sustain a cardiac arrest outside of the hospital.

\section{Introduction}

The crucial role of Cardiopulmonary Resuscitation (CPR) in the event of Sudden Cardiac Arrest (SCA) has been undebatable for years. CPR has an indisputable effect on the outcome and prognosis of a patient sustaining a SCA; this is of outmost importance when considering the large pool of patients undergoing either an In-Hospital Cardiac Arrest (IHCA) or an Out-Of-Hospital Cardiac Arrest (OHCA). In the UK approximately 35.000 patients sustain an IHCA every year. ${ }^{1}$ Data regarding OHCA suggest that its prevalence is very high both in Europe and in the USA. ${ }^{2}$ Return Of Spontaneous Circulation (ROSC) is a crucial indicator of the effectiveness of the interventions made when treating a SCA. However, in a setting of OHCA even though one third of patients achieve ROSC when treated, survival rate is only $8 \%{ }^{3}$ Also, even though almost half of IHCA adult victims achieve ROSC initially, longterm survival is also poor. ${ }^{4}$

Effective Chest Compressions (CCs) are the cornerstone of high-quality CPR. Optimal CCs are characterized by a proper rate and adequate depth, minimal interruptions and full chest recoil. ${ }^{5}$ However, in a resuscitation setting the delivery of optimal CCs can be highly demanding for the rescuer, their quality being disproportional to operator exhaustion. In a study of manual CCs, over $80 \%$ of rescuers experienced serious back discomfort, mostly related to the duration of CPR and a significant $20 \%$ suffered back injury or prolapsed disc. ${ }^{6}$

Also special considerations on the quality of CCs have to be taken into account when SCA happens in non-friendly settings, such as inside the moving ambulance, the cardiac CT suite or even the cardiac catheterization laboratory; in the latter, the extreme danger by the ionizing radiation to the person providing CCs must be taken seriously into account. In an attempt to resolve this issue, Automated Chest Compression Devices (ACCDs) have been implemented in the resuscitation process. ACCDs deliver constantly high level compressions, of consistent rate and depth over even prolonged periods of time; as a side note, they run on batteries and can last up to one hour when disconnected from power supply. ${ }^{7,8}$ There are two mechanism types of ACCDs, based on the compressions delivery mode: (i) the one using a Piston-Driven technique (PD) (Lucas, Life-Stat, Weil) - (mostly well-known and used is the Lucas device - Figure 1 - Stryker Corp., Sweden), thus applying antero-posterior energy on the sternum; (ii) the other uses a LoadDistribution Band (LDB) (Autopulse - Figure 2 - ZOLL Medical 
Corp., USA) which distributes the force applied on the torso more evenly. ${ }^{9}$ Studies have compared manual CCs and ACCDs in both OHCA and in IHCA. In this review, we attempt to present up to date literature regarding their role in cardiac arrest, application in clinical practice and current guidelines.

\section{Application in IHCA}

Extracorporeal Membrane Oxygenation (ECMO), mainly percutaneous veno-arterial ECMO, and left ventricular assist devices as means of advanced CPR in the hospital setting have been proved to have favourable outcomes; however they are considerably invasive, very expensive and therefore are not widely or universally used. The major concern during CPR is the delivery of uninterrupted CCs, as well as the effectiveness of the resuscitation team in performing all interventions needed for a successful outcome. A further concern in places like the catheterization laboratory is the extensive exposure of the staff performing CCs in the detrimental effects of ionizing radiation.

Mechanical devices can provide a suitable solution to all these challenges. ${ }^{10}$ Research so far has been mainly focused on the use of ACCDs in the pre-hospital setting. Their use in the IHCA setting has not been studied as thoroughly, despite of the fact that it engulfs advantages that otherwise lack. More specifically, in IHCA contrary to OHCA, the devices are deployed more quickly and

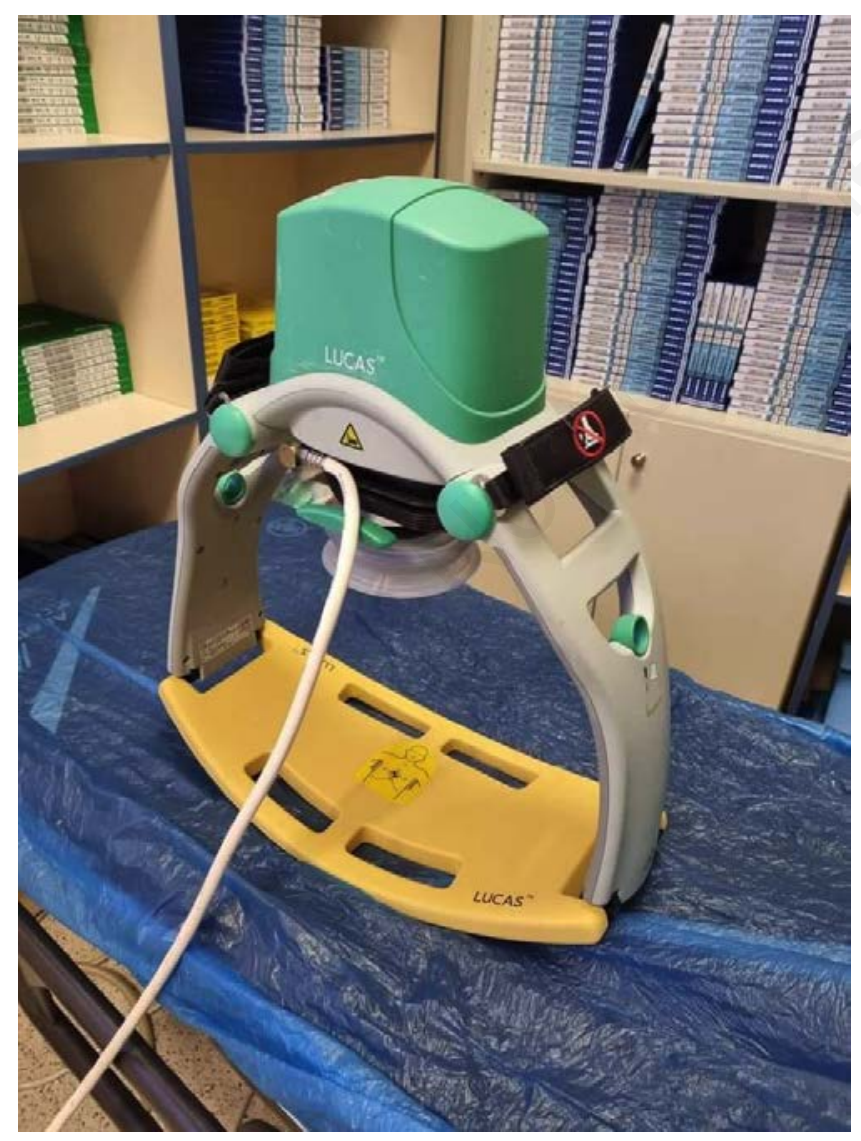

Figure 1. The Lucas device (piston-driven device for mechanical chest compressions): a piston pushes downwards the sternum at a constant rate 100 per minute, instead of a human resuscitator. effectively during the arrest. In addition, although most of cardiac arrests occur outside of the hospital environment, hospital stuff is more frequently exposed to cardiac arrest cases thus having developed greater experience. ${ }^{1}$ A systematic ACCD review including trials, case reports and case series reported, with the exception of two studies, a survival rate of $39 \%$, and of those who survived, a full neurological recovery in $91 \% .{ }^{11}$ Both types of ACCDs where used (piston and band). Those results were attributed to the early initiation of mechanical CCs along with uninterrupted and consistent delivery of high-quality compressions, especially in patients with a reversible cause of cardiac arrest. ${ }^{11}$ Similarly, a meta-analysis of nine studies (of which three were randomised), including both types of devices, reported improved hospital and 30-day survival rates with the use of ACCDs. Regarding short-term survival, reports suggested good outcomes with the use of ACCDs. However, there were limitations, including lack of reports regarding neurological outcomes and CPR quality. The risk of bias in the observational studies and the indirectness of evidence were also highlighted. ${ }^{1}$ Patient safety was also examined; rib fractures, a sternal fracture and one liver laceration were reported in two of the studies analysed, with no report of which device used in each case. Injuries, however, were similar between ACCDs and manual CCs. ${ }^{1}$ A forensic study identified as an Autopulse pattern of injuries the following: posterior rib fractures, skin abrasions along the anterolateral chest and shoulder, vertebral fractures, and few visceral injuries. $^{12}$ A two-year Autopulse registry for IHCA, reported favourable outcomes, expressed as survival to discharge, when the device was used in cardiac arrest of reversible causes. ${ }^{13}$ In the same case-series, however, four different situations leading to Autopulse failure on four different patients where mentioned, including battery depletion, clip detachment, compression band twist and difficult backboard placement. The need of correct placement in order to avoid iatrogenic injuries is strongly highlighted. ${ }^{13}$

Better in-hospital ACCDs outcomes can be attributed not only to specialized staff and better time allocation, but also to the advanced infrastructure of the hospital environment, ranging from airway support including intubation and ventilation during CPR, to acute patient care including drugs, induced hypothermia, catheterization and intensive care unit monitoring post-resuscitation.

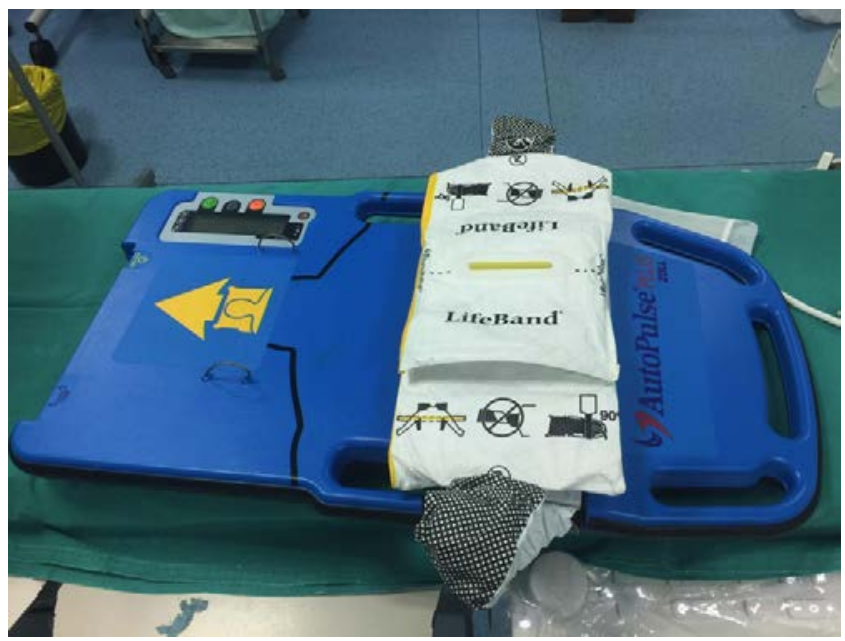

Figure 2. The Autopulse device (load-distributing band device). A band rhythmically constricts the entire rib cage, pumping the heart at a rate of 80 compressions per minute. 
Quality of manual CCs in the hospital pose a challenge, as in-hospital patient mattresses tend to absorb up to $40 \%$ of the force produced during compressions. ${ }^{14}$ On the other hand, the time pause needed for the deployment of the device is considered a considerable limitation in IHCAs. However, data imply that trained resuscitation teams deploy the devices effectively with a minimum pause in ongoing CPR efforts. ${ }^{15}$ The learning curve required for the successful deployment of an ACCD is, however, brought into attention when implementing mechanical CCs into the CPR protocol. ${ }^{13}$

Recently, the design of a trial that randomized victims in a $3: 1$ ratio (mechanical CPR: manual CPR) was published. ${ }^{16}$ It attempted to examine the outcomes of a PD device (Lucas) use during resuscitation of IHCA victims with non-shockable initial rhythms, but ended up rather identifying important limitations regarding an efficient study design that would successfully implement mechanical CPR into the resuscitation protocol. More specifically, hospital survival was lower than anticipated, possibly due to late intraarrest randomization, non-superior CC quality in the Lucas arm and low overall recruitment; the authors highlight the feasibility of such a successful trial design only when all limitations and challenges will be taken into consideration. ${ }^{16}$ Cardiac arrest in an emergency department setting, although typically classified as an IHCA entity, comprises mainly of OHCA patients, thus tending to be a separate entity. Therefore it is categorized in trials as such. ${ }^{17,18}$

\section{Application in the cardiac catheterization laboratory}

Historic data of more than 20 years ago stated that approximately $1.3 \%$ of all patients undergoing cardiac catheterization sustained a cardiac arrest. ${ }^{19}$ In the modern era, despite complex percutaneous coronary and structural interventions (PCI, TAVI etc), improved techniques and equipment led to a fall in SCA prevalence. ${ }^{20}$ ROSC is often achieved fast, due to early defibrillation and prompt initiation of CPR by experienced nursing and medical cathlab personnel. However, prolonged CPR may be required; in this case fluoroscopy equipment and table height may pose challenges in manual CCs application..$^{20-23}$ Furthermore, exposure of the staff to high doses of ionizing radiation during CPR in the catheterization laboratory is a major concern, as high doses of radiation accumulated over time have been associated with malignancies, skin reactions, cataract, bone injuries and heritable effects in the descendants. ${ }^{21}$ Thus, protection against uncontrolled exposure to radiation has been established through radiation safety program that implements radiation monitoring, protective shields, training of personnel and modernized fluoroscopy equipment..$^{22}$ The use of ACCDs has been studied and case reports, citing the use of the Lucas device, have highlighted its advantages; the most prominent is the lack of need for additional staff for compressions and the ability of ongoing life-saving interventions (for example primary percutaneous coronary intervention in STEMIs) along with the effective, uninterrupted use of the device which is translucent and can even continue compressions with extremely minimal pause for defibrillation. ${ }^{24-27}$ With the Lucas device, all views except for straight antero-posterior are applicable during cardiac catheterization, and the device permits free movement of the X-ray detector. ${ }^{28}$ The force delivered does not affect the catheterization itself, although minor interruptions for coronary stent deployment may be needed. ${ }^{28}$ On the other hand, the delay of the initial deployment has been reported as a drawback. However, with training, this time can be reduced to a median of seven seconds. ${ }^{9}$ Data so far have contemplated the use of an ACCD during PCI, in most cases with the Lucas device, and suggested improved ROSC rates along with better survival rate and neurological outcomes at hospital dis- charge compared to manual CCs. ${ }^{29}$ Furthermore, a study further supported the improved ROSC rate even in patients that were brought to the laboratory with ongoing ACCDs compressions. ${ }^{23}$ Mechanical compression devices can bridge patients in cardiac arrest to deployment of a fully percutaneous mechanical circulatory support in the cathlab. However, survival to hospital discharge was similar to manual mechanical CCs. ${ }^{23}$ In a case series, use of the Lucas device was found to be beneficial for patients that sustained cardiac arrest during catheterization, especially during PCI, ${ }^{28}$ a finding attributed to the vital blood pressure levels the device can effectively maintain. ${ }^{26}$ Use of band distributor ACCDs (Autopulse) has also been reported, also allowing interventions in the cathlab and providing uninterrupted compressions during PCI. ${ }^{30,31}$ Continuous effective CPR in a sterile field along with unobstructed visualization of the coronary arteries during catheterization were therefore observed with the use of Autopulse in the cathlab. ${ }^{13}$ Based on the above, the American Heart Association in its 2010 Guidelines for Cardiopulmonary Resuscitation ${ }^{32}$ stated that mechanical piston or load-distributing band chest compression devices may be considered in patients undergoing PCI or CT scans, for prolonged resuscitation (class IIa) or when manual resuscitation is difficult (class IIb). It is mandatory that trained personnel implement the use of such devices. However, up to this point, there is insufficient evidence to support or refute their routine use in cardiac arrest. The European Resuscitation Council (ERC) supported and co-authored the 2010 guidelines $^{32}$ and in the recent 2015 guidelines once again strongly recommended the use of ACCDs in the cathlab during coronary interventions. ${ }^{33}$

\section{Application in OHCA}

Data regarding ACCD use in a setting of OHCA are more extensive than IHCA. Although animal models have suggested favourable outcomes with mechanical CPR, ${ }^{34,35}$ real-life application on cardiac arrest patients has produced mixed results. An early randomized trial concluded that the use of a load-distributing band is associated with worse neurological outcomes as well as worse 4hour survival, compared to manual CCs. ${ }^{36}$ Long pause for its application has been noted with a piston device, a finding that could possibly explain its unfavourable outcomes. ${ }^{37}$ On the other hand, a more recent meta-analysis, that examined both types of devices in an OHCA setting regarding ROSC in a total of twelve studies, showed that mechanical CPR benefits outcome, with the condition that the staff applying it is sufficiently trained. ${ }^{38}$ Minimal interruptions of compressions achieved through the ACCD allows rescuers to perform other activities, crucial to patient survival. Interestingly, in this meta-analysis load-distribution devices outperformed piston-driven devices, while piston-driven devices exhibited similar ROSC rates to manual CCs. ${ }^{38}$ Thirty-day survival, survival to hospital admission or survival to discharge were comparable between manual CCs and the Lucas device in another meta-analysis, although manual CPR proved superior to Autopulse; in terms of patient safety, manual CCs were superior to the devices. ${ }^{39}$ The use of ACCDs in OHCA has been categorized in three different subsections, depending on the setting in which the resuscitation is taking place.

\section{Use in the Emergency Room}

SCA cases treated within emergency departments around the world are cases of OHCA as well as patients that suffer cardiac arrest while waiting in the emergency room (ER). ${ }^{17} \mathrm{~A}$ randomized trial examined the effect of trained personnel using an LDB device 
protocol in the ER and concluded that it provides better CPR quality to the patient, with shorter interruption during deployment of the device. ${ }^{15}$ Similarly, the use of the Autopulse device in two ERs in Singapore revealed an association between mechanical CPR period and ROSC rates after adjusted analyses, but no association with other outcomes..$^{40}$ On the other hand, a randomized trial executed in Japan showed that the arm that was treated for OHCA with a device in the ER had worse survival rates to hospital discharge along with worse admission to hospital survival and decreased likelihood of ROSC, possibly due to deployment pauses in CCs and lack of mechanical CPR quality feedback. ${ }^{41}$ Differences in findings among studies may be attributed to different protocols, different study populations or risk of bias. ${ }^{17}$

\section{Use during transfer to hospital}

Worldwide, SCA patients are more and more often transferred to the hospital with ongoing CPR, unfortunately of doubtful quality. ${ }^{42,43}$ Patients may arrest during transport or OHCA patients may need transportation. ${ }^{44,45}$ Manual compressions during transfer to the ED have been proved to be both ineffective for high-quality CPR and unsafe for emergency medical service (EMS) staff. ${ }^{46}$ Various difficulties - including but not limited to - uneven pavements and tight doorways during transfer of the victim to the ambulance, sudden stops, accelerations, turns and confined ambulance space, adversely affect CPR quality. ${ }^{44}$ Mechanical compressions exhibit advantages, such as non-stop continuousness of compressions, while all EMS staff is seated during transportation. ${ }^{38}$ Furthermore, an observational study found that ACCDs minimize compression interruptions that occur with manual CPR during extrication of a patient, except for the deployment pause. ${ }^{47}$ In a case-control study, higher rates of ROSC were reported in the mechanical CPR arm. ${ }^{48}$ Same results were reported in an observational study, that confirmed higher ROSC rates and survival to hospital admission when a device was implemented in CPR during transportation. ${ }^{49}$ In a review on OHCA patients that received mechanical CPR during transfer to the hospital revealed better CPR quality for mechanical CPR regarding rate, compression ratio and depth, irrespective to vehicle type and transportation conditions. ${ }^{44}$ Both types of devices were examined. However, in regard to survival and outcomes, the heterogeneity of the included trials challenged the generalization of the results. Consequently, it appeared that mechanical and manual CPR may have similar outcomes; however, the importance of high-quality CPR and proper personnel training in both cases is highlighted throughout. ${ }^{44,50}$

Mechanical compression devices can be available in ambulances to support victims of OHCA during transportation, in case cardiac arrest re-occurs. This is especially the case when a long distance needs to be covered. The Danish cardiac arrest registry (41.186 OHCA patients in Denmark, during 2001-2013), ${ }^{50}$ showed a marked reduction in mortality when resuscitated out-of-hospital arrest victims were transferred to coronary angiography capable tertiary hospitals versus transfer to the nearest district hospital (odds ratio 0.78 , with $95 \%$ confidence interval $0.76-0.81$ ) and especially when they underwent emergency PCI or coronary bypass surgery. This favorable effect of the so-called "invasive" hospitals persisted, irrespective of the distance the resuscitated victim had to travel by ambulance (odds ratio 0.93, 0.97, 0.98, 0.99 and 1.01 for transfers of $0-5,5-10,10-20,20-50$ and 50-100 kilometers compared to 101-200 kilometers, respectively). ${ }^{50}$

\section{Routine deployment on scene}

In a large proportion of OHCA, CPR in general and chest compressions in particular are initiated by bystanders. ${ }^{2}$ Data from
European registries report a variable percentage of ROSC, depending on first rhythm, witnessed SCA, ongoing CPR and differences in EMS systems. ${ }^{2,3}$ In a randomized study examining effects of mechanical compressions by a PD device in OHCA, no difference was noted in early survival between device and manual CCs. ${ }^{51}$ The pause of compressions during manual CPR needed to deliver a defibrillation shock has been proved to be an independent predictor of survival in OHCA. ${ }^{52}$ ACDDs can offer continuous high-quality compressions, with full chest recoil, on the scene of cardiac arrest, while defibrillation can take place simultaneously. ${ }^{38}$ However, in the randomized ASPIRE trial the deployment of the load-distribution device costs a delay of 2.1 minutes to first shock in ventricular fibrillation; due to neurological and survival adverse outcomes the trial was prematurely terminated. ${ }^{36}$ Prolongation of time to first shock was prominent in CIRC and LINC trials as well. ${ }^{53,54}$ Rescuers were trained, in an attempt to minimize interruptions in both those studies. Furthermore, cost-effectiveness does not favor the routine use of mechanical CPR in OHCA. ${ }^{55}$ Factors such as body weight and applicability of the device have also been highlighted, as not all patients can receive properly a device; thus, further prolongation of application may occur. ${ }^{54}$ Last, patient safety is not yet fully clarified, as very few studies have addressed the issue. ${ }^{56}$ In this randomized trial, liver lacerations associated with massive hemorrhage were reported in two patients in the Lucas arm along with one Autopulse-associated tension pneumothorax that caused an air embolus; manual CC-associated hazards were fewer. ${ }^{56}$ Pre-hospital use of ACCDs has been shown to offer worse neurological outcomes to hospital discharge when compared to manual CPR. ${ }^{57,58}$ The LINC trail randomized on the scene SCA victims into manual compression versus Lucas-mediated compressions. Primary outcome was four-hour survival after ROSC. The results of the trial revealed no differences in neither four-hour survival between the two arms, nor the six-month survival and neurological outcomes. Although defibrillation took place in all victims during compressions irrespective of initial rhythm, in the Lucas arm first defibrillation shock occurred 1.5 minutes later. The device well-suited $95 \%$ of the patients. ${ }^{54}$ The effects of on-scene Lucas deployment were examined by the PARAMEDIC trial as well. This was a 2:1 randomized trial (manual:Lucas) that presented its results as part of a meta-analysis, alongside two other studies. In the primary outcome of thirty-day survival, no superiority of the device was proven. ${ }^{59}$ In the randomized CIRC trial a LDB device versus manual CPR was examined. Survival to hospital discharge was examined as the primary outcome, while CPR quality was throughout measured. The two arms of the study displayed similar survival rates and neurological outcomes to hospital discharge. However, the manual arm received controlled and optimal CPR, although this may not be always the case in a real-life OHCA scenario. ${ }^{53}$ Serious adverse events were similar between the LINC and CINC trials. The heterogeneity of trials conducted for comparison of techniques poses a challenge when techniques are being compared. ACCDs have been proposed to improve pre-hospital and admission to hospital survival especially when used by a two member paramedic team, when the victim is young in age and when the arrest takes place in the town center. ${ }^{8}$ A 2019 meta analysis did not support the use of LUCAS in OHCA settings regarding clinical outcomes compared with manual chest compression. ${ }^{60}$

\section{Current guidelines and the COVID-19 era}

Routine use of ACCDs is not recommended according to cur- 
rent guidelines. ${ }^{32,61}$ However, in special cases, such as during transportation or catheterization, during prolonged $\mathrm{CPR}$ or CPR taking place in a confined space, their use is advised. ${ }^{61-63}$ Furthermore, consensus documents along with guidelines support their use during PCI or CT (class IIa). ${ }^{20,64}$ As aforementioned, the American Heart Association, in its 2010 Guidelines for Cardiopulmonary Resuscitation, ${ }^{32}$ classified the use of ACCDs for prolonged resuscitation as class IIa in patients undergoing PCI or CT scans. In the case of difficult manual resuscitation, their use was categorized as class IIb. Only trained personnel must handle the devices. Up to that point, they stated that there was insufficient evidence to either support or refute their routine use in cardiac arrest. ERC coauthored the 2010 guidelines ${ }^{32}$ and later strongly recommended the use of ACCDs in the cathlab during coronary interventions in its 2015 guidelines. ${ }^{33}$

The need for minimal interruptions is highlighted, restricted only to the initial deployment of the device. In an ambulance staffed by two paramedics, a mechanical device can be proven useful, although guidelines do not fully support it. ${ }^{65}$

The use of mechanical CPR in special settings has been studied as well. It has been used as a bridge to uncontrolled organ donation. ${ }^{66,67}$ Although ethical and clinical challenges are raised, the use of mechanical CPR can reduce the time of warm ischemia, ${ }^{68}$ vital in a setting of uncontrolled organ harvest. ${ }^{17}$ In another setting, during insertion of extracorporeal CPR (cardiopulmonary bypass) the use of an ACCD may be proven useful and it is applied with positive results, although data are still limited. ${ }^{69-71}$ It is of outmost importance that the ethical dilemmas and clinical decisions made in a case of refractory SCA require very careful consideration. ${ }^{17,72}$

During the SARS-Cov2 virus pandemic (COVID-19), new questions arose regarding CPR and new protocols were implemented in patient management. Modifications were installed by the $\mathrm{ERC}^{73}$ and also by various national societies ${ }^{74}$ in the proposed $\mathrm{CPR}$ process (basic and advanced life support algorithm) to protect rescuers from cross-infection. In a setting of OHCA, a piece of cloth is placed on the victim's face before the initiation of CPR while the rescuer is advised to wear a mask and wash his/her hands immediately after. In the case of IHCA, the advanced life support algorithm is followed with extreme caution to personal protective equipment, airway management and compressions. The use of ACCDs is advised, as soon as the device is available. ${ }^{74,75}$

\section{Conclusions}

Data regarding the use of mechanical CPR in cases of both outof- and in-hospital cardiac arrest are mixed. In general, the use of devices in OHCA situations seems to show weak and conflicting evidence on the outcomes of the victims, while in IHCA it has a rather positive impact. High-quality CPR remains the key to a successful resuscitation and as such its importance is highlighted in all studies comparing manual and mechanical CPR. Large registries and randomized trials should further elucidate this debate, CPR quality measurement being a key component in their design.

\section{References}

1. Couper K, Yeung J, Nicholson T, et al. Mechanical chest compression devices at in-hospital cardiac arrest: A systematic review and meta-analysis. Resuscitation 2016;103:24-31.

2. Gräsner JT, Lefering R, Koster RW, et al. EuReCa ONE-27
Nations, ONE Europe, ONE Registry: A prospective one month analysis of out-of-hospital cardiac arrest outcomes in 27 countries in Europe. Resuscitation 2016;105:188-95.

3. Gräsner JT, Wnent J, Herlitz J, et al. Survival after out-of-hospital cardiac arrest in Europe - Results of the EuReCa TWO study. Resuscitation 2020;148:218-26.

4. Schluep M, Gravesteijn BY, Stolker RJ, et al. One-year survival after in-hospital cardiac arrest: A systematic review and meta-analysis. Resuscitation 2018;132:90-100.

5. Kleinman ME, Brennan EE, Goldberger ZD, et al. Part 5: Adult basic life support and cardiopulmonary resuscitation quality: 2015 American Heart Association guidelines update for cardiopulmonary resuscitation and emergency cardiovascular care. Circulation 2015;132:S414-35.

6. Jones AYM. Can cardiopulmonary resuscitation injure the back? Resuscitation 2004;61:63-7.

7. Yuksen C, Prachanukool T, Aramvanitch K, et al. Is a mechanical-assist device better than manual chest compression? A randomized controlled trial. Open Access Emerg Med 2017;9:637.

8. Kłosiewicz T, Puślecki M, Zalewski R, et al. Impact of automatic chest compression devices in out-of-hospital cardiac arrest. J Thorac Dis 2020;12:2200-27.

9. Levy M, Yost D, Walker RG, et al. A quality improvement initiative to optimize use of a mechanical chest compression device within a high-performance CPR approach to out-ofhospital cardiac arrest resuscitation. Resuscitation 2015;92:327.

10. Couper K, Smyth M, Perkins GD. Mechanical devices for chest compression: To use or not to use? Curr Opin Crit Care 2015;21:188-94.

11. Lameijer H, Immink RS, Broekema JJ, Ter Maaten JC. Mechanical cardiopulmonary resuscitation in in-hospital cardiac arrest: a systematic review. Eur J Emerg Med 2015;22:379-83.

12. Pinto DC, Haden-Pinneri K, Love JC. Manual and automated cardiopulmonary resuscitation (CPR): A comparison of associated injury patterns. J Forensic Sci 2013;58:904-9.

13. Spiro JR, White S, Quinn N, et al. Automated cardiopulmonary resuscitation using a load-distributing band external cardiac support device for in-hospital cardiac arrest: A single centre experience of AutoPulse-CPR. Int J Cardiol 2015;180:7-14.

14. Perkins GD, Kocierz L, Smith SCL, et al. Compression feedback devices over estimate chest compression depth when performed on a bed. Resuscitation 2009;80:79-82.

15. Ong MEH, Quah JLJ, Annathurai A, et al. Improving the quality of cardiopulmonary resuscitation by training dedicated cardiac arrest teams incorporating a mechanical load-distributing device at the emergency department. Resuscitation 2013;84:508-14.

16. Couper K, Quinn T, Booth K, et al. Mechanical versus manual chest compressions in the treatment of in-hospital cardiac arrest patients in a non-shockable rhythm: A multi-centre feasibility randomised controlled trial (COMPRESS-RCT). Resuscitation 2021;158:228-235. Epub 2020 Oct 7.

17. Poole K, Couper K, Smyth MA, Yeung J, Perkins GD. Mechanical CPR: Who? When? How? Crit Care 2018;22:140.

18. Tan SC, Leong BSH. Cardiac arrests within the emergency department: An Utstein style report, causation and survival factors. Eur J Emerg Med 2018;25:12-7.

19. Webb JG, Solankhi NK, Chugh SK, et al. Incidence, correlates, and outcome of cardiac arrest associated with percutaneous coronary intervention. Am J Cardiol 2002;90:1252-4. 
20. Latsios G, Mpompotis G, Tsioufis K, et al. Advanced cardiopulmonary resuscitation (CPR) in the Catheterization Laboratory: Consensus document of the Working Groups of 1) Cardiopulmonary Resuscitation/Acute Cardiac Care and 2) Hemodynamic and Interventional Cardiology, Hellenic Cardiological Society. Hell J Cardiol 2017;58:396-400.

21. Hirshfeld JW, Ferrari VA, Bengel FM, et al. 2018 ACC/HRS/NASCI/SCAI/SCCT Expert Consensus Document on Optimal Use of Ionizing Radiation in Cardiovascular Imaging: Best Practices for Safety and Effectiveness: A Report of the American College of Cardiology Task Force on Expert Consensus Decision Pathways. J Am Coll Cardiol 2018;71:e283-e351.

22. Chambers CE, Fetterly KA, Holzer R, et al. Radiation safety program for the cardiac catheterization laboratory. Catheter Cardiovasc Interv 2011;77:546-56.

23. Venturini JM, Retzer E, Estrada JR, et al. Mechanical chest compressions improve rate of return of spontaneous circulation and allow for initiation of percutaneous circulatory support during cardiac arrest in the cardiac catheterization laboratory. Resuscitation 2017;115:56-60.

24. Grogaard HK, Wik L, Eriksen M, et al. Continuous Mechanical Chest Compressions During Cardiac Arrest to Facilitate Restoration of Coronary Circulation With Percutaneous Coronary Intervention. J Am Coll Cardiol 2007;50:1093-4.

25. Agostoni P, Cornelis K, Vermeersch P. Successful percutaneous treatment of an intraprocedural left main stent thrombosis with the support of an automatic mechanical chest compression device. Int J Cardiol 2008;124:e19-21.

26. Larsen AI, Hjørnevik ÅS, Ellingsen CL, Nilsen DWT. Cardiac arrest with continuous mechanical chest compression during percutaneous coronary intervention. A report on the use of the LUCAS device. Resuscitation 2007;75:454-9.

27. Yadav K, Truong HT. Cardiac Arrest in the Catheterization Laboratory. Curr Cardiol Rev 2018;14:115-20.

28. Wagner H, Terkelsen CJ, Friberg H, et al. Cardiac arrest in the catheterisation laboratory: A 5-year experience of using mechanical chest compressions to facilitate PCI during prolonged resuscitation efforts. Resuscitation 2010;81:383-7.

29. Wagner H, Hardig BM, Rundgren M, et al. Mechanical chest compressions in the coronary catheterization laboratory to facilitate coronary intervention and survival in patients requiring prolonged resuscitation efforts. Scand J Trauma Resusc Emerg Med 2016;24:4.

30. Latsios G, Antonopoulos A, Vogiatzakis N, et al. Successful primary PCI during prolonged continuous cardiopulmonary resuscitation with an automated chest compression device (AutoPulse). Int J Cardiol 2016;225:258-9.

31. Dallan LA, Vargas TT, Janella BL, et al. Chest compressions using mechanical devices are more effective than manual compressions in cardiac arrest concomitant with emergency percutaneous coronary intervention. J Am Coll Cardiol 2014;63:A1868.

32. Field JM, Hazinski MF, Sayre MR, et al. Part 1: Executive summary: 2010 American Heart Association Guidelines for Cardiopulmonary Resuscitation and Emergency Cardiovascular Care. Circulation 2010;122:S640-56.

33. Truhlár A, Deakin CD, Soar J, et al. European Resuscitation Council Guidelines for Resuscitation 2015. Section 4. Cardiac arrest in special circumstances. Resuscitation 2015;95:148201.

34. Halperin HR, Paradis N, Ornato JP, et al. Cardiopulmonary resuscitation with a novel chest compression device in a porcine model of cardiac arrest: Improved hemodynamics and mechanisms. J Am Coll Cardiol 2004;44:2214-20.

35. Ikeno F, Kaneda H, Hongo Y, et al. Augmentation of tissue perfusion by a novel compression device increases neurologically intact survival in a porcine model of prolonged cardiac arrest. Resuscitation 2006;68:109-18.

36. Hallstrom A, Rea TD, Sayre MR, et al. Manual chest compression vs use of an automated chest compression device during resuscitation following out-of-hospital cardiac arrest: A randomized trial. JAMA 2006;295:2620-8.

37. Yost D, Phillips RH, Gonzales L, et al. Assessment of CPR interruptions from transthoracic impedance during use of the LUCASTM mechanical chest compression system. Resuscitation 2012;83:961-5.

38. Westfall M, Krantz S, Mullin C, Kaufman C. Mechanical versus manual chest compressions in out-of-hospital cardiac arrest: A meta-analysis. Crit Care Med 2013;41:1782-9.

39. Khan SU, Lone AN, Talluri S, et al. Efficacy and safety of mechanical versus manual compression in cardiac arrest - A Bayesian network meta-analysis. Resuscitation 2018;130:1828.

40. Hock Ong ME, Fook-Chong S, Annathurai A, et al. Improved neurologically intact survival with the use of an automated, load-distributing band chest compression device for cardiac arrest presenting to the emergency department. Crit Care 2012;16:R144.

41. Hayashida K, Tagami T, Fukuda T, et al. Mechanical cardiopulmonary resuscitation and hospital survival among adult patients with nontraumatic out-of-hospital cardiac arrest attending the emergency department: A prospective, multicenter, observational study in Japan (SOS-KANTO [Survey of Survivos after Out-of-Hospital Cardiac Arrest in Kanto Area] 2012 Study). J Am Heart Assoc 2017;6:e007420.

42. Olasveengen TM, Wik L, Steen PA. Quality of cardiopulmonary resuscitation before and during transport in out-of-hospital cardiac arrest. Resuscitation 2008;76:185-90.

43. Russi CS, Myers LA, Kolb LJ, et al. A Comparison of Chest Compression Quality Delivered During On-Scene and Ground Transport Cardiopulmonary Resuscitation. West J Emerg Med 2016;17:634-9.

44. Ong MEH, Mackey KE, Zhang ZC, et al. Mechanical CPR devices compared to manual CPR during out-of-hospital cardiac arrest and ambulance transport: A systematic review. Scand J Trauma Resusc Emerg Med 2012;20:39.

45. Tazarourte K, Sapir D, Laborne FX, et al. Refractory cardiac arrest in a rural area: Mechanical chest compression during helicopter transport. Acta Anaesthesiol Scand 2013;57:71-6.

46. Wik L, Kramer-Johansen J, Myklebust H, et al. Quality of cardiopulmonary resuscitation during out-of-hospital cardiac arrest. JAMA 2005;293:299-304.

47. Lyon RM, Crawford A, Crookston C, et al. The combined use of mechanical CPR and a carry sheet to maintain quality resuscitation in out-of-hospital cardiac arrest patients during extrication and transport. Resuscitation 2015;93:102-6

48. Casner M, Andersen D, Isaacs SM. The impact of a new CPR assist device on rate of return of spontaneous circulation in out-of-hospital cardiac arrest. Prehospital Emerg Care 2005;9:61-7.

49. Hock Ong ME, Ornato JP, Edwards DP, et al. Use of an automated, load-distributing band chest compression device for out-of-hospital cardiac arrest resuscitation. JAMA 2006;295:2629-37. 
50. Tranberg T, Lippert FK, Christensen EF, et al. Distance to invasive heart centre, performance of acute coronary angiography, and angioplasty and associated outcome in out-of-hospital cardiac arrest: A nationwide study. Eur Heart J 2017;38:164552.

51. Smekal D, Johansson J, Huzevka T, Rubertsson S. A pilot study of mechanical chest compressions with the LUCASTM device in cardiopulmonary resuscitation. Resuscitation 2011;82:702-6.

52. Cheskes S, Schmicker RH, Christenson J, et al. Perishock pause: An independent predictor of survival from out-of-hospital shockable cardiac arrest. Circulation 2011;124:58-66.

53. Wik L, Olsen JA, Persse D, et al. Manual vs. integrated automatic load-distributing band CPR with equal survival after out of hospital cardiac arrest. The randomized CIRC trial. Resuscitation 2014;85:741-8.

54. Rubertsson S, Lindgren E, Smekal D, et al. Mechanical chest compressions and simultaneous defibrillation vs conventional cardiopulmonary resuscitation in out-of-hospital cardiac arrest: The LINC randomized trial. JAMA 2014;311:53-61.

55. Marti J, Hulme C, Ferreira Z, et al. The cost-effectiveness of a mechanical compression device in out-of-hospital cardiac arrest. Resuscitation 2017;117:1-7.

56. Koster RW, Beenen LF, Van Der Boom EB, et al. Safety of mechanical chest compression devices AutoPulse and LUCAS in cardiac arrest: A randomized clinical trial for non-inferiority. Eur Heart J 2017;38:3006-13.

57. Buckler DG, Burke RV, Naim MY, et al. Association of Mechanical Cardiopulmonary Resuscitation Device Use with Cardiac Arrest Outcomes: A Population-Based Study Using the CARES Registry (Cardiac Arrest Registry to Enhance Survival). Circulation 2016;134:2131-3.

58. Youngquist ST, Ockerse P, Hartsell S, Stratford C, Taillac P. Mechanical chest compression devices are associated with poor neurological survival in a statewide registry: A propensity score analysis. Resuscitation 2016;106:102-7.

59. Perkins GD, Lall R, Quinn T, et al. Mechanical versus manual chest compression for out-of-hospital cardiac arrest (PARAMEDIC): A pragmatic, cluster randomised controlled trial. Lancet 2015;385:947-55.

60. Liu M, Shuai Z, Ai J, Tang K, et al. Mechanical chest compression with LUCAS device does not improve clinical outcome in out-of-hospital cardiac arrest patients: A systematic review and meta-analysis. Medicine (Baltimore) 2019;98:e17550

61. Soar J, Nolan JP, Böttiger BW, et al. European Resuscitation Council Guidelines for Resuscitation 2015. Section 3. Adult advanced life support. Resuscitation 2015;95:100-47.

62. Brooks SC, Anderson ML, Bruder E, et al. Part 6: Alternative techniques and ancillary devices for cardiopulmonary resuscitation: 2015 American Heart Association guidelines update for cardiopulmonary resuscitation and emergency cardiovascular care. Circulation 2015;132:S436-43.

63. Wang PL, Brooks SC. Mechanical versus manual chest compressions for cardiac arrest. Cochrane Database of Systematic Reviews 2018:CD007260

64. Neumar RW, Shuster M, Callaway CW, et al. Part 1: Executive summary: 2015 American Heart Association guidelines update for cardiopulmonary resuscitation and emergency cardiovascular care. Circulation 2015;132:S315-67.

65. Dabrowski M, Sip M, Dabrowska A, et al. It is impossible to follow the ERC algorithm in a two-paramedics ambulance team. Resuscitation 2017;118:E43.

66. Ortega-Deballon I, Hornby L, Shemie SD. Protocols for uncontrolled donation after circulatory death: A systematic review of international guidelines, practices and transplant outcomes. Crit Care 2015;19:268.

67. Mateos-Rodríguez A, Pardillos-Ferrer L, Navalpotro-Pascual JM, et al. Kidney transplant function using organs from nonheart-beating donors maintained by mechanical chest compressions. Resuscitation 2010;81:904-7.

68. Manara AR, Murphy PG, Ocallaghan G. Donation after circulatory death. Br J Anaesth 2012;108:i108-21.

69. Yannopoulos D. The interventional cardiologist as a resuscitator: a new era of machines in the cardiac catheterization laboratory. Hell J Cardiol 2017;401-2.

70. Lamhaut L, Tea V, Raphalen JH, et al. Coronary lesions in refractory out of hospital cardiac arrest (OHCA) treated by extra corporeal pulmonary resuscitation (ECPR). Resuscitation 2018;126:154-9.

71. Stub D, Bernard S, Pellegrino V, et al. Refractory cardiac arrest treated with mechanical CPR, hypothermia, ECMO and early reperfusion (the CHEER trial). Resuscitation 2015;86:88-94.

72. Dalle Ave AL, Shaw DM, Gardiner D. Extracorporeal membrane oxygenation (ECMO) assisted cardiopulmonary resuscitation or uncontrolled donation after the circulatory determination of death following out-of-hospital refractory cardiac arrest-An ethical analysis of an unresolved clinical dilemma. Resuscitation 2016;108:87-94

73. Nolan JP, Monsieurs KG, Bossaert L, et al. European Resuscitation Council COVID-19 guidelines executive summary. Resuscitation 2020;153:45-55.

74. Latsios G, Synetos A, Mastrokostopoulos A, et al. Cardiopulmonary Resuscitation in patients with suspected or confirmed Covid-19. A Consensus of the Working group of the Cardiopulmonary Resuscitation of the Hellenic Society of Cardiology. Hell J Cardiol 2020;S1109-9666(20)30200-1.

75. Chahar P, Marciniak D. Cardiopulmonary resuscitation in COVID-19 patients. Cleve Clin J Med 2020; Online ahead of print. 